\title{
SUPPORTING EMERGENCE OF THREADED LEARNING CONVERSATIONS THROUGH AUGMENTING INTERACTIONAL AND SEQUENTIAL COHERENCE
}

\author{
CSCL Conference 2003
}

\begin{abstract}
In this article, we propose a structure of threaded learning conversations taking place in virtual learning communities. This new structure aims at facilitating the emergence of learning conversations in these learning communities. These threaded conversations take place in a forum-type tool that uses a tree data structure composed of threads to organize group communication. We identify two situations which discourage the emergence of the learning conversations taking place in threaded based tools. We are interested in, namely "interactional incoherence" and "sequential incoherence". For each of these identified drawbacks, we conceive mechanisms which intend to surmount these incoherencies: first, the localization of topics in a message, based on the "what you answer is what you link" criteria; and second, a visualization that allows merging in a single view the time order and the thread order of the messages. Finally, we present a tool based on these mechanisms and describe some preliminary results of its use.
\end{abstract}

\section{INTRODUCTION}

From a general point of view, this work takes place in a research project which aims at contributing to a better understanding of interactions within virtual learning communities, in the context of computer-based tools. We design, implement and test innovative tools to provide data that will enable us to progress in our conceptualization of our research domain and phenomena. This way, the tool we build from the analysis described in this article takes part in our research project, as a means of providing an environment that facilitates the emergence of a special type of social interaction: the learning conversations that taking place in virtual learning community. These interactions will be analyzed later, as part of the mentioned research project (being out of the scope of this paper) through social network analysis methods.

This paper focuses on the research issue of supporting learning conversations taking place in "forum-type" tools. The expression "forum-type" is used for a mainly text-based and asynchronous electronic conferencing system that makes use of a tree hierarchical data structure of enchained messages, called threads. We will call threaded conversations, the conversations taking place in these forum-type tools. In this work, the notion of "supporting" goes in hand with the creation of strategies from which the students support themselves and each other: we are not advocating the creation of automated systems that support the student activity. 
The objective of this paper is to create strategies in order to facilitate the emergence and development of these learning conversations. The creation of these strategies is based on methodology described by Winograd (Winograd \& Flores, 1986). Winograd and Flores have proposed a design method of analyse for human activity domains, starting from the analysis of "breakdowns" in these domains. A breakdown is an "interrupted moment of our habitual, standard, being-in-theworld". They state that the design is only necessary in activities where a recurring structure of breakdown emerges: "the design must envisage the forms of rupture and provide a space of possibilities for the action when those are produced". As we will see in the following sections, the domain of threaded conversations sticks closely to this recurrent breakdown pattern definition. Therefore, we study some of the breakdowns in threaded conversations which discourage the learning conversation emergence. As our intention is to establish mechanisms that contribute to this emergence, we propose a new structure of threaded conversations taking place in these "forum-type" tools.

In this article we concentrate on two difficulties or breakdowns that the use of these tools raise for the emergence and development of learning conversations: Firstly, the fact that the current forum-type systems have an interactional incoherence derived from the difference between message unity which users can handle and the one they can reference. This incoherence causes information loss and reference confusions; Secondly, the fact that current forum-type systems carry a sequential incoherence caused by the disruption between the temporal order of messages and the thread order of messages.

In the proposed tool the participants have the capacity of reacting to topics in the messages by choosing them through the criteria "what you answer is what you link" (WYAIWYL). It is important to note that we do not use semantic text analysis methods for locating the topics, but the participants themselves choose each topic.

This paper is organized as follows. First comes an overview of the supporting learning conversation domain. We then identify the breakdowns or difficulties which arise in the utilisation of "forum-type" tools for emergence of learning conversations. Then we describe our proposals to overcome the problems mentioned. And finally, we present our prototype that builds these proposals and present some results from an empirical study.

\section{SUPPORTING LEARNING CONVERSATIONS}

Learning is fundamentally a social activity, as described by the theory of the communities of practices (Lave \& Wenger, 1991) and the socio constructivist approach (Staarman, De Laat, \& Van der Meijden, 2002). In these approaches, it is understood that much of the actual learning happens through learning conversations.

The learning conversations are the ones that "go further than just realizing information exchange; rather, they allow participants to make connections between previously unrelated ideas, or to see old ideas in a new way. They are conversations that lead to conceptual change" (R. Bellamy, 1997). 
We found a large number of research focused on the technological support to encourage learning conversations (Baker, De Vries, \& Lund, 1999; Hoadley, Hsi, \& Berman, 1995; Quignard \& Baker, 1999). Generally, this research trends have an approach based on the labelling (through sentence openers) of the participant's contributions, and in this way encourage some defined types of conversation. In this approach, various authors have created different sentence openers' structures, to obtain a specific structure of conversation. CONNECT is an example of such system developed by Baker et al (Baker et al., 1999). CONNECT is a tool to encourage the specific (epistemic) interactions by partial structuring, where students are asked to categorize their opinions with respect to sentences of their partners. Another work following this approach is the Speakeasy interface (Hoadley et al., 1995). Speakeasy is a structured discussion tool for learning. A structuring feature of this tool is the ability to label predefined links between messages ("And", "Or", "But", "Question" and "Summary") to indicate the link relationship. Hoadley, His et al. argue these link labels establish a relational context between contributions.

In a less structured approach, we find Vapillon's work (Vapillon, 2000). Although his objective isn't the encouragement of learning conversations (he has created a structured discussion tool for coordination), his system allows users to define the threads from parts of messages. He also takes a structured approach by creating a categorization of the interactions (co-orientation, anti-orientation, interrogation and others), where the participants are asked to categorize their answers. These approaches lie on the idea of encoding speakers' intentions into categories, and, in this way, structuring the conversation and the dynamics of organizational interaction over time and creating specific interactive learning mechanisms (Quignard \& Baker, 1999).

In this paper, we take a more flexible approach by developing a tool that intends to facilitate the emergence and development of learning conversation through mechanisms in which the users are not forced to categorize any of their interactions. This choice is based on the following arguments. First, the particular context of our research project: virtual learning communities, as some newsgroups or on-line forums (e.g. http://slashdot.org/). In these virtual communities, different types of interactions take place. Reducing them (e.g. only epistemic interactions), by using categorization and sentence openers can be a factor preventing the development of these communities. Second, we adhere to the idea that (Suchman, 1994) the definition of categories for the contributions, instead of contributing to the encouragement of some specific conversations or interactions, actually is a political approach for the reproduction of an established social order (See (Suchman, 1994) for a detailed criticism related to the use of sentence categorization approaches).

\section{BREAKDOWNS IN THREADED LEARNING CONVERSATIONS}

In virtual learning communities, as said, almost all the learning happens through the learning conversations that develop over communication tools like forums, newsgroups, or other written media of asynchronous communication. Using these tools, the development of conversations is necessarily shaped by the underlying 
technological hierarchical structure, in this case, threads. But carrying out a conversation through this fixed structure, introduces some breakdowns.

We particularly concentrate on two specific problems, which relate to our interest in facilitating the emergence and development of the learning conversations: interactional incoherence and sequential incoherence.

\subsection{Interactional incoherence}

Several authors have observed that within a message we can find several topics that appear and develop as threads (Condon \& Cech, Forthcoming; Vapillon, 2000). This situation is caused by the fact that usually the messages that users receive are answered by choosing the part of the message that users want to answer. This segmentation can be done by the selection of a part of the message or by the multiple segmentation of a message (by answering several paragraphs). However, this segmentation is not visible in the actual threads of messages, which only denote the link relation between the messages, without taking into account the part or parts of the message selected by the users. In this way, the topics are not easily localized because they are buried in the rest of the message contents.

We conceptualize this problem by introducing the concept of granularity of exchanges. The concept of granularity of exchanges goes in hand with the notion of minimal unity of exchange we can manipulate and reference. If we analyze the forum-type tools under this perspective, we realize that there is a difference between the manipulation granularity and the reference granularity of the messages. This difference originates from the fact that users can manipulate the messages segmenting them into topics, by answering only parts of a message, but they can't create the references for these selected parts of a message because the references in the forum-type tools are only between messages. Consequently, the threads in the forum-type tools hide the true relations between the topics. This difference between manipulation granularity and reference granularity is what we call interactional incoherence.

The interactional incoherence sets two problems for the emergence of learning conversations: Firstly, the topic definition is an important element for encouraging learning conversations (R. K. E. Bellamy \& Woolsey, 1988). However, forum-type tools do not allow this localization capability, as these tools hide the topics raised by the participants in the whole message when referencing it. Secondly, research has shown (R. K. E. Bellamy \& Woolsey, 1988) that the establishment of a common ground encourages learning conversations. However, research has also shown that forum-type tools force users to maintain a specific structure of communication imposed by the underlying technology used which prevents the establishment of the common ground (McCarthy, Miles, \& Monk, 1991). This way the forum-type tools structure, in relation to its capacity to establish a common ground makes the emergency of learning conversations difficult.

In this context a tool with a more flexible structure, where the participants can define their threads from more specific topics, can facilitate the constitution of a common ground. 


\subsection{Sequential incoherence}

Several authors have observed a dissociation in threaded conversations between the temporal order of messages and the thread order of messages (Davis \& Rouzie, 2002; Herring, 1999). This dissociation is caused by the fact that in most threaded visualization tools, the factors of space and time are dislocated. This way, in forumtype tools, users have the capacity to look at the same conversation from two different viewpoints: messages ordered by time, and thread-ordered messages view.

This dislocation makes conversations not as seamless as they should: sorting by threads makes the tracking of timely exchanges difficult. In this sense Davis and Rouzie (Davis \& Rouzie, 2002) note " as messages can be added to any node in a thread at any later date, students often failed to follow the development of a particular debate"; Also, sorting messages by time doesn't allow regarding the actual placement of a message in the threads.

All these difficulties have direct consequences on collaborative learning and the learning conversations on which it is based. This dislocation prevents the participants building their own "adequate mental representation of the virtual space and time within which they interact" (Pincas, 2001). So students have a weak perception of the global discussion, since "in order to communicate and learn collaboratively in such an environment, participants need to engage in what they can perceive as a normal discussion" (Pincas, 2001).

\section{AUGMENTING COHERENCE: PROPOSED STRATEGIES}

In order to facilitate the emergence of learning conversations, we conceived two strategies to deal with the interactional and sequential incoherence problems in thread-based conversation tools.

Regarding interactional incoherence, we propose changing the actual minimal unity of exchange (the message) for a new minimal unity, the topics which are in the messages. This change will allow us to reference any segment of the message and so to track down easily every participant's interventions throughout his interactions. The localization is realized through the "What You Answer Is What You Link" (WYAIWYL) criteria. This way, the participants, in order to respond to a topic, must select it. The tool will create a link between the selected topic and the answer. Therefore, threaded conversations can be defined for the users from the selected topics. It is important to note that we do not use semantic text analysis methods for locating the topics; topics are defined by the participants.

Regarding sequential incoherence, we propose a visualization of the threads which allows visualizing quickly and graphically the flow and patterns of online discourse at the same moment in time. We will merge both types of existing views (the time order and the thread order of messages) in a single graph like visualization.

Contrasted with a common threaded conversation tool (like forum or newsgroups), this proposed tool increases the interactional coherence by facilitating topic location in the conversation. It also increases the sequential coherence, by allowing to connect message precedence (time adjacency) and patterns of threads (turn taking adjacency) in a same visualization. 


\section{PROTOTYPE IMPLEMENTATION}

In this section, we illustrate and explain the implementation of our prototype. Students interact with the prototype via its user interface to browse, read, reply and create messages. This prototype is an electronic tool that allows communication for an electronic learning community. Members of the learning group participate in the exchange of information and viewpoints on issues related to their interest.

Some characteristics of the system can be seen in the browser space (the top of the window of the figure 1), where users can navigate through discussion threaded messages, by means of its graph-based visual environment. In this modality of visualization, both proposed mechanisms which aim at facilitating the emergence and development of learning conversations are implemented.

\subsection{Prototype Interface}

Figure 1 illustrates the interface of the prototype. The top of the window display is the browser space, the space in which the learners browse the messages which will be shown in the workspace -the window at the bottom. In the workspace, learners inspect, reply and create messages.

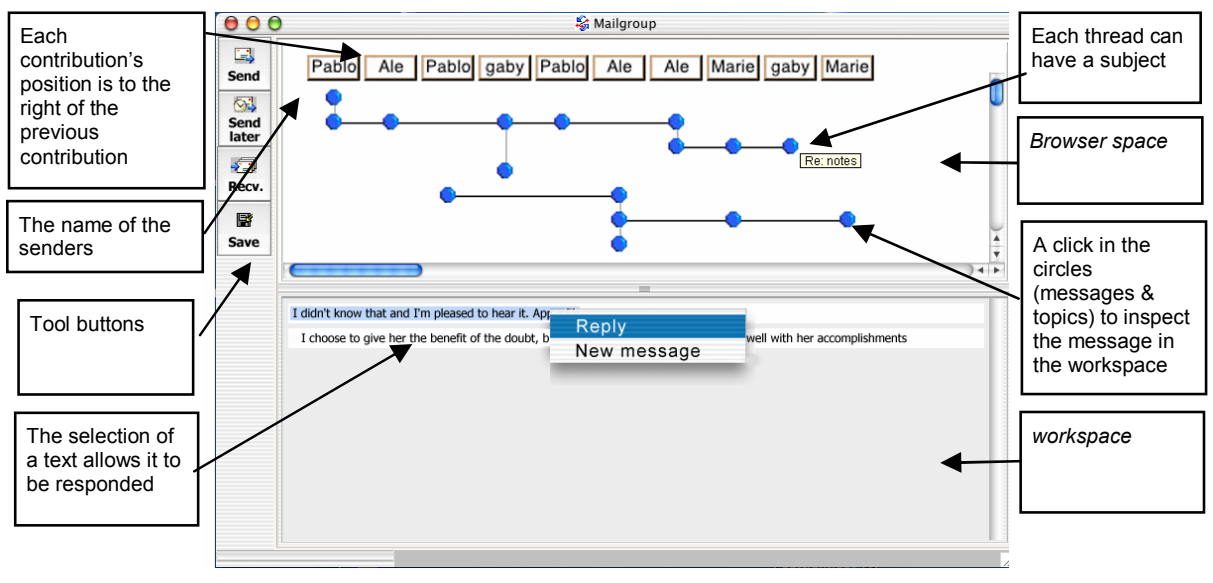

Figure 1. Interface of the developed tool

The system generates the graphical visualization from the answers and new topics created by the users.

The browser space shows the whole of the discussion represented by a graph: Vertexes -the circles in figure 1-correspond to the topics of a conversation, and the edges correspond to the links between the topics. The lines placed between the vertexes of different columns correspond to the links explicitly created by the users between the two topics-. The tree structures of the browser space are interactive, 
since users can click on the vertexes and look at the topics contents in the working space.

\subsection{Dynamics of threaded conversation construction}

The construction of the threaded conversation is based on the participants' reactions to the messages. Here, the users have the capacity to segment the messages as they please, by choosing the topics in which they want to react with the desired granularity.

Figure 2 illustrates the relationship between the actions of the participants in the workspace, and the representation of their actions in the browser space. Particularly, this diagram represents a situation where a user (participant B) answers part of a message (a specific topic) from participant A. Figure 2.(1) shows the participant A message's contents as user B sees it in the workspace. The message is displayed in the browser space just as a vertex placed at the lower part of sender name. Figure 2.(2) illustrates: First, an action of participant B who reacts to one part of participant A's message by selecting it and answering through a contextual menu; Second, the effect of this action in the browser space. As a result, a new column is added to the right-hand side. Now, the original message shows two connected vertexes with a vertical line: one corresponding to a selected part of a message and, consequently, related to the answer of participant $\mathrm{B}$. And the other vertex, corresponding to the rest of participant A's message (i.e. the part that hasn't been answered by anybody). The presence of this vertex can appear disconcerting, since it really doesn't correspond to a topic (as it has not been responded), but its presence contributes to make visible the texts in messages that have not been reacted on.

\begin{tabular}{|c|c|c|c|}
\hline & Workspace & Browser space & \\
\hline (1) & $\begin{array}{l}\text { How about: an accurate } \\
\text { perception of reality. } \\
\text { We have to first observe } \\
\text { reality in order to } \\
\text { perceive it. If reality } \\
\text { is subjective, then [...] }\end{array}$ & A & $\begin{array}{l}\text { Representation } \\
\text { of Message } \\
\text { from participant } \\
\text { A }\end{array}$ \\
\hline (2) & $\begin{array}{l}\text { How about: an accurate } \\
\text { perceptinn af roslitw } \\
\text { We have Reply } \\
\text { reality New message } \\
\text { perceiv tc. 1. Icurtcy } \\
\text { is subjective, then [...] }\end{array}$ & A & 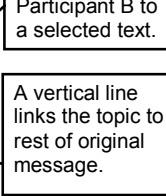 \\
\hline
\end{tabular}

Figure 2. One can visualizes two steps -2.(1) and 2.(2) -of the answering message dynamics.

\section{EMPIRICAL STUDY AND PRELIMINARY RESULTS}

An empirical study was designed in order to collect feedback on the actual characteristics of our prototype from the user's perspective. In this study, eight 
groups of 4 or 5 students were recruited. The students corresponded to second year undergrad, who voluntarily carried out, during one month, a distance collaborative activity. During this study, the tool was used just as the medium of communication and discussion, not as a point of concern in itself. The students' activity objective was, actually, to carry out a collaborative evaluation of several educational software packages from a given list of criteria. We didn't want the students to be discussing the tool, nor thinking about it. We were interested in evaluating just how they could "work it out". The figure 4 illustrates the browser space of the prototype, which reveals a real group discussion in an advanced state.

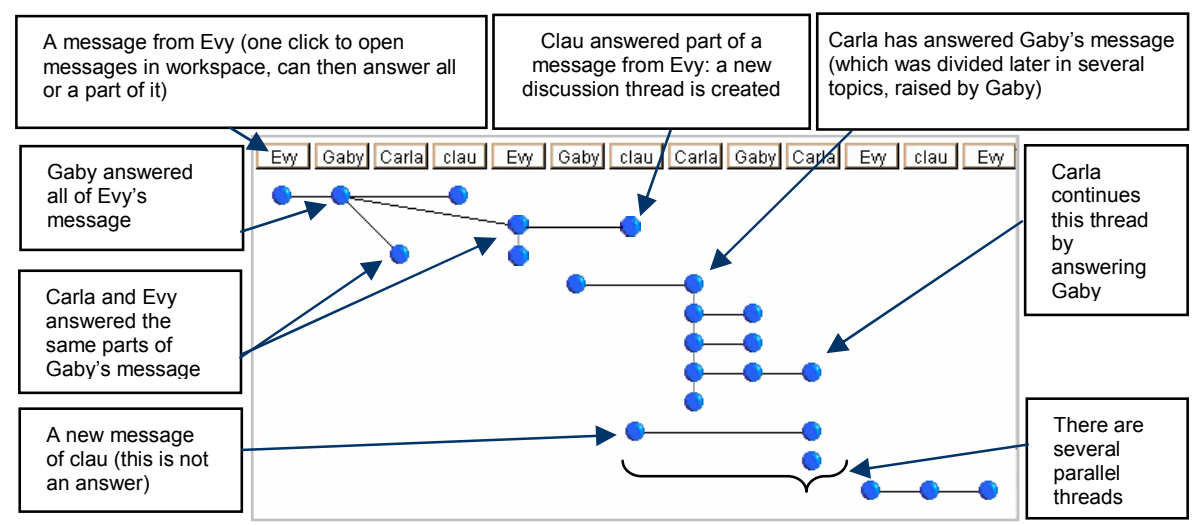

Figure 3.Illustrates the browser space, which reveals a real group discussion in an advanced state.

The discussion contributions were studied in order to examine the impact of the new thread visualization model in the threaded conversations of the participants in a learning context.

Preliminary results showed that students largely used the new capability of selecting parts of a message, over the classical approach of answering the whole message. As the students could choose more granular and "findable" topics in the easily navigable threads view, they could organize and collaborate better.

The use of this tool produced the collateral effect of organizing the speech of the students' messages in paragraphs. They indeed affirm that creating the messages this way will make it easier for other participants to locate and respond to parts of a message (topics), helping to the topic visibility and definition.

On the other hand, the proposed visualization produced, when students located several topics in a message, larger topic segmentation, which contributed to the dispersion of conversation in small units. This situation can increase the fragmentation problem of traditional threaded conversations (Hewitt, 2001).

Regarding the proposed interface, we found some interface usability problems associated with the graph-like visualization (and not a standard text-based interface). Users take some time to get used to this new visualization. Also, the ambiguity of message and topic visualization: some participants interpreted incorrectly the 
meaning of certain graphical cues, specially the decomposition of a message into topics. This last problem can be repaired designing a clear interface difference between messages and topics.

\section{CONCLUSIONS AND FURTHER WORKS}

Although research has shown an amount of difficulties associated with forum-type tools for its educational utilization (Herring, 1999; Hewitt, 2001; Vapillon, 2000), these tools actually are widely used in this domain: they are used in computer conferencing packages, web-based newsgroups and e-learning systems. This paper presents a tool that proposes strategies to overcome two specific breakdowns found in these actually widespreadly used educational threaded conversation tools.

Even though this tool can be used in other contexts than learning communities, we argue that the best benefits of this new structure can be drawn from these communities, considering that it is in this context that the creation of a "common ground" is more critical to help virtual or distant learning conversations emerge and develop.

This tool introduces a change in the granularity of the interactions and new dynamics of answering based on the principle WYAIWYR ("what you answer is what you link"). These characteristics allow going from a thread-fixed structure to a more flexible and granular structure, that considers the aspects of interactions' automanagement through the users capacity of defining the topics and their development.

The tool presented in this work focuses on the notion of enhancing coherence in threaded conversations for facilitating the emergence and development of learning conversations. Beyond these characteristics of the tool, and in our research project's context, it represents the instrument from which we start with our analysis of interactions based on social network algorithms.

\{pablo.reyes, pierre.tchounikine\}@lium.univ-lemans.fr (LIUM, France).

\section{REFERENCES}

Baker, M., De Vries, E., \& Lund, K. (1999). Designing computer-mediated epistemic interactions. Paper presented at the Artificial Intelligence in Education, Le Mans.

Bellamy, R. (1997). Support for Learning Conversations. Retrieved 11/12/2002, 2002, from http://kmi.open.ac.uk/seminars/seminars-text.cfm.

Bellamy, R. K. E., \& Woolsey, K. (1988). Learning Conversations. SIGCHI Bulletin, 30(2).

Condon, S. L., \& Cech, C. G. (Forthcoming). Discourse Management in Three Modalities. In S. Herring (Ed.), Computer-Mediated Conversation: Hampton Press.

Davis, M., \& Rouzie, A. (2002). Cooperation vs. Deliberation: Computer Mediated Conferencing and the Problem of Argument in International Distance Education. International Review of Research in Open and Distance Learning, 3(1).

Herring, S. (1999). Interactional Coherence in CMC. Paper presented at the 32nd Hawai'i International Conference on System Sciences, Hawai.

Hewitt, J. (2001). Beyond threaded discourse. International Journal of Educational Telecommunications, $3(7), 207-221$. 
Hoadley, C. M., Hsi, S., \& Berman, B. P. (1995). The Multimedia Forum Kiosk and SpeakEasy. Paper presented at the ACM Multimedia, San Francisco, CA.

Lave, J., \& Wenger, E. (1991). Situated Learning: Legitimate Peripheral Participation. Cambridge, UK: Cambridge University Press.

McCarthy, J. C., Miles, V. C., \& Monk, A. F. (1991). An experimental study of common ground in textbased communication. Paper presented at the Human factors in computing systems: reaching through technology, CHI'91.

Pincas, A. (2001). E-learning by virtual replication of classroom methodology. Paper presented at the The Humanities and Arts higher education Network, HAN

Quignard, M., \& Baker, M. (1999). Favouring computer mediated argumentative dialogue in problem solving situations. Paper presented at the Artificial Intelligence in Education, Le Mans.

Staarman, J. K., De Laat, M., \& Van der Meijden, H. (2002). Seeking attunement in collaborative learning. Paper presented at the Fifth Congress of the International Society for Cultural Research and Activity Theory, Amsterdam.

Suchman, L. (1994). Do Categories Have Politics? The Language /Action Perspective Reconsidered. Computer Supported Cooperative Work (CSCW), 3(2), 177-190.

Vapillon, J. (2000). Contribution à l'étude de la conversation dans le cadre du travail coopératif assisté par ordinateur. Paris XI University, Paris.

Winograd, T., \& Flores, F. (1986). Understanding Computers and Cognition: Ablex, Norwood. 